\title{
Acute allergic interstitial nephritis after use of pantoprazole
}

\author{
Jeffrey Ricketson BSc, Gil Kimel MD MSc, James Spence MD, Rene Weir MD
}

The case: A 57-year-old man presented to hospital with a 2-week history of progressive malaise, myalgia, fever, nausea, vomiting, diarrhea, polyuria and polydipsia. He had a history of gastresophageal reflux that did not respond to ranitidine. He had started taking oral pantoprazole $(40 \mathrm{mg}$ daily) 6 weeks earlier. The patient had a history of coronary artery disease and was taking acetylsalicylic acid (81 mg daily) and atorvastatin (40 mg daily). He reported having taken ibuprofen $(400 \mathrm{mg})$ for myalgia no more than 3 times over the 2-week period before presentation. He had no history of renal disease or drug allergies.

On presentation, the patient was afebrile (temperature $36.5^{\circ} \mathrm{C}$ ), with a blood pressure of $127 / 82 \mathrm{~mm} \mathrm{Hg}$, a pulse of 72 beats/min, a respiratory rate of 18 breaths $/ \mathrm{min}$ and an oxygen saturation of $97 \%$ in room air. The jugular venous pressure was measured at the sternal angle. His chest sounds were normal, and there was no pericardial friction rub or peripheral edema. The results of a dermatological examination were unremarkable.

The results of laboratory tests are listed in Table 1. Of note, the patient had a serum creatinine level of 300 (nor-

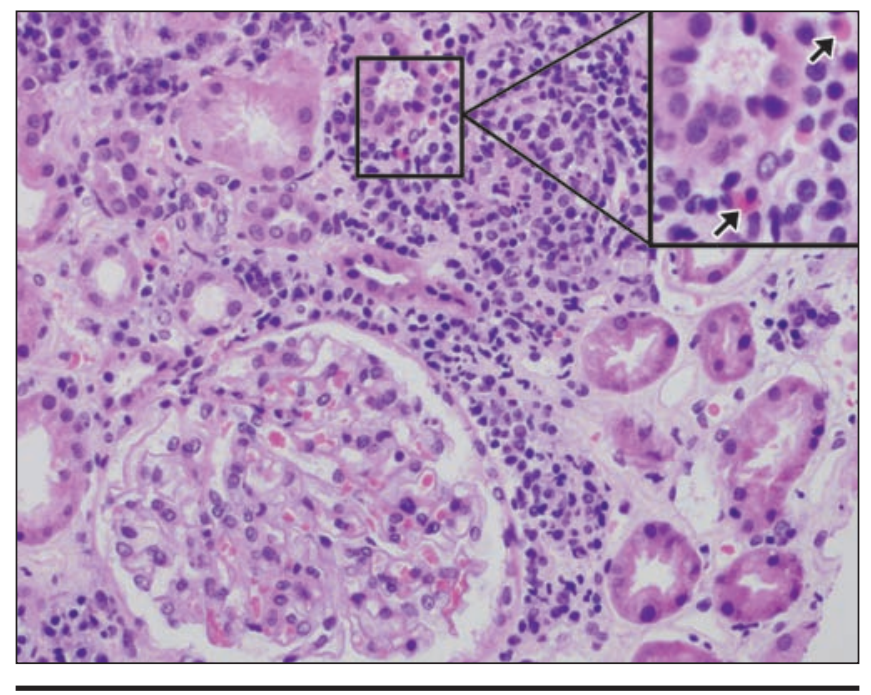

Figure 1: A needle core biopsy of the patient's kidney, showing inflammatory process predominantly in the interstitium (hematoxylin-eosin stain, original magnification $\times 40$ ). Insert shows a higher magnification, with arrows pointing to eosinophils in the interstitium.

\section{Key points}

- Acute interstitial nephritis should be considered in cases of acute renal failure if pre- and post-renal causes have been ruled out.

- There are numerous causes of acute interstitial nephritis, including adverse drug reactions (e.g., to antimicrobials, nonsteroidal anti-inflammatory drugs and acid suppressors), infections and systemic diseases.

- Treatment is initially directed at the systemic disease or removing the offending agent.

- Steroid therapy may be indicated in cases of acute interstitial nephritis that do not respond to initial treatment.

- Timely diagnosis is important because early intervention improves the outcome.

mal 53-106) $\mu \mathrm{mol} / \mathrm{L}$. Two years ago, his creatinine level had been $100 \mu \mathrm{mol} / \mathrm{L}$. He had mild hyperkalemia, but his electrolyte levels were otherwise normal. His leukocyte and eosinophil counts were normal. The ratio of protein to creatinine in his urine was 28 (normal $0-23$ ), and the protein level in a 24-hour urine collection was 410 (normal < 150) mg. Protein electrophoresis showed that the protein in the urine was predominantly albumin. When analyzed by use of Wright stain, the first urine sample was negative for eosinophils, but $1 \%$ of the leukocytes in the second sample were eosinophils.

Treatment included volume repletion and discontinuation of pantoprazole. Ultrasonography showed that both of the patient's kidneys were of normal size, and that there was normal echogenicity with no hydronephrosis. Over the next 4 days, his creatinine level decreased to $235 \mu \mathrm{mol} / \mathrm{L}$. A needle core biopsy of 1 of the patient's kidneys was performed on day 6 . The biopsy was indicated because the patient had ongoing acute renal failure with major proteinuria for which pre- and post-renal causes had been ruled out (there had been very minor improvement with volume repletion and the results of a renal ultrasound were normal). The biopsy showed moderate-to-severe patchy interstitial infiltrates, predominantly plasma cells with some lymphocytes and occasional eosinophils (Figure 1). Immunoflourescence was negative for $\lg G$, IgA, IgM, C3, C1q and fibrin antibodies. These results supported the diagnosis of acute interstitial nephritis.

From the Faculty of Medicine, University of British Columbia, Vancouver, BC 
The patient was prescribed high-dose oral prednisone ( $1 \mathrm{mg} / \mathrm{kg}$ daily) with stepwise tapering. After 45 days, the patient's creatinine level had decreased and plateaued at $145 \mu \mathrm{mol} / \mathrm{L}$. Prednisone therapy was discontinued on day 98 . On day 168 , his creatinine level was $147 \mu \mathrm{mol} / \mathrm{L}$, there was no proteinuria, and his blood pressure was normal.

Esophagogastroduodenoscopy was performed to evaluate the patient's reflux disease. The results suggested severe esophagitis with a benign lower esophageal ulcer. The patient was given both ranitidine and domperidone,

Table 1: Results of laboratory investigations

\begin{tabular}{|c|c|}
\hline Laboratory test (normal value) & Result* \\
\hline \multicolumn{2}{|l|}{ Hematology } \\
\hline Hemoglobin (136-170 g/L) & $131 \mathrm{~g} / \mathrm{L}$ \\
\hline Leukocytes $\left(4.5-11.0 \times 10^{9} / \mathrm{L}\right)$ & $9.6 \times 10^{9} / \mathrm{L}$ \\
\hline Neutrophils $\left(2.05-7.05 \times 10^{9} / L\right)$ & $6.02 \times 10^{9} / \mathrm{L}$ \\
\hline Eosinophils $\left(0-0.44 \times 10^{9} / L\right)$ & $0.37 \times 10^{9} / \mathrm{L}$ \\
\hline Platelets $\left(150-400 \times 10^{9} / L\right)$ & $474 \times 10^{9} / L$ \\
\hline Creatinine $(53-106 \mu \mathrm{mol} / \mathrm{L})$ & $300 \mu \mathrm{mol} / \mathrm{L}$ \\
\hline Urea (1.2-3.0 mmol/L) & $14.4 \mathrm{mmol} / \mathrm{L}$ \\
\hline Albumin (35-55 g/L) & $29 \mathrm{~g} / \mathrm{L}$ \\
\hline \multicolumn{2}{|l|}{ Chemistry } \\
\hline Sodium (135-145 mmol/L) & $138 \mathrm{mmol} / \mathrm{L}$ \\
\hline Potassium (3.5-5.0 mmol/L) & $5.2 \mathrm{mmol} / \mathrm{L}$ \\
\hline Chloride (101-11 mmol/L) & $104 \mathrm{mmol} / \mathrm{L}$ \\
\hline Bicarbonate (24-32 mmol/L) & $25 \mathrm{mmol} / \mathrm{L}$ \\
\hline \multicolumn{2}{|l|}{ Urine testing } \\
\hline Leukocytes ( 0 cells/hpf) & 40-100 cells/hpf \\
\hline Red blood cells ( 0 cells/hpf) & 6 cells/hpf \\
\hline Granular casts ( 0 casts/lpf) & 3 casts/lpf \\
\hline Eosinophils (negative) & $\begin{array}{c}\text { First sample negative, } 1 \% \\
\text { of leukocytes in second } \\
\text { sample }\end{array}$ \\
\hline $\begin{array}{l}\text { Cultures (bacterial, tuberculosis) } \\
\text { (negative) }\end{array}$ & Negative \\
\hline $\begin{array}{l}\text { 24-hour urine protein } \\
(<150 \mathrm{mg} / \text { day })\end{array}$ & $410 \mathrm{mg} / \mathrm{day}$ \\
\hline $\begin{array}{l}\text { Ratio of protein to creatinine } \\
(0-23)\end{array}$ & 28 \\
\hline Urine sodium (mmol/L) & $49 \mathrm{mmol} / \mathrm{L}$ \\
\hline Urine creatinine (mmol/L) & $2.9 \mathrm{mmol} / \mathrm{L}$ \\
\hline \multicolumn{2}{|l|}{ Rheumatology } \\
\hline $\begin{array}{l}\text { Antineutrophil cytoplasmic } \\
\text { antibodies (negative) }\end{array}$ & Negative \\
\hline Antinuclear antibodies (negative) & Negative \\
\hline $\begin{array}{l}\text { Serum electrophoresis for } \\
\text { paraprotein (negative) }\end{array}$ & Negative \\
\hline $\begin{array}{l}\text { Urine electrophoresis for } \\
\text { paraprotein (negative) }\end{array}$ & Negative \\
\hline
\end{tabular}

Note: hpf = high-power field, lpf = low-powered field.

*Abnormal results are in bold. and he was instructed not to take any proton pump inhibitors or nonsteroidal anti-inflammatory drugs, except for acetylsalicylic acid (81 mg daily). As of the patient's last visit, his reflux was controlled with ranitidine (300 mg twice daily) and calcium carbonate tablets ( $750 \mathrm{mg}$ twice daily).

Our patient was diagnosed with acute interstitial nephritis likely caused by the use of pantoprazole. The key features leading to this diagnosis were acute renal failure from a renal cause with associated proteinuria and eosinophiluria, as well as a renal biopsy that showed interstitial inflammation and a lack of evidence for other causes of renal failure.

Acute interstitial nephritis is responsible for $1 \%-3 \%$ of cases of acute renal failure. ${ }^{1}$ It occurs following injury to the tubules or interstitium of the kidney. The common causes of acute interstitial nephritis are listed in Box 1 . Acute interstitial nephritis may be suspected in cases of acute renal failure where pre- and post-renal causes have been ruled out.

\section{Clinical characteristics}

The clinical presentation of acute interstitial nephritis can vary; however, patients typically present with general symptoms of renal failure, such as nausea, vomiting and fatigue. Injury to the medulla can inhibit the ability to concentrate urine, leading to polyuria and polydipsia. ${ }^{2}$ Hypertension and edema are not typical features; these signs can help distinguish acute interstitial nephritis from a vascular or glomerular problem. ${ }^{2}$ Laboratory investigations typically show elevated levels of creatinine (Box 2). Depending on the extent of tubular involvement, abnormalities in electrolyte levels may be present, the most common of which are hyponatremia and hyperkalemia. ${ }^{2}$ Pyuria and proteinuria are almost always present, and hematuria is present in $90 \%$ of cases. ${ }^{3}$ With druginduced acute interstitial nephritis, eosinophiluria occurs in about $80 \%$ of cases. ${ }^{3}$

To diagnose acute interstitial nephritis, one must first rule out pre- and post-renal causes of acute renal failure and investigate the potential causes of acute interstitial nephritis. The suspicion of acute interstitial nephritis should be increased if kidney function improves after withdrawal of the suspected offending agent. The diagnosis can be confirmed by subsequent resolution of kidney function as shown by creatinine levels. In cases of severe renal failure or persistent loss of kidney function after removing the suspected agent, a biopsy is required to confirm the diagnosis of acute interstitial nephritis and to rule out other diseases that have a similar presentation, such as acute tubular necrosis. ${ }^{2}$ Because acute interstitial nephritis is potentially life-threatening, accurate and timely diagnosis of this condition is critical.

\section{Causes of acute interstitial nephritis}

Drug reactions are the most frequent cause of acute interstitial nephritis. The most common drugs that cause acute interstitial nephritis are penicillins, cephalosporins, sulfon- 
amides and nonsteroidal anti-inflammatory drugs. ${ }^{1}$ The mechanism that causes acute interstitial nephritis is unknown, but it is thought to be an immunologic effect, for which there are no known risk factors. ${ }^{4}$ The onset of acute interstitial nephritis can occur from hours to months after beginning the inciting agent; about $80 \%$ of patients will show renal symptoms within 3 weeks (average 10 days). ${ }^{3}$ Along with the clinical manifestations of acute interstitial nephritis, these patients may also present with low-grade fever, maculopapular rash and mild arthralgia. ${ }^{3}$ The diagnosis is more difficult to make in the case of long-term therapy with a drug that can cause acute interstitial nephritis, such as phenytoin or allopurinol, and when the delay in development of the disease is months rather than weeks. Up to $40 \%$ of patients with drug-induced acute interstitial nephritis may have persistently elevated levels of creatinine, reflecting irreversible kidney damage. ${ }^{3}$

The mainstay treatment of drug-induced acute interstitial nephritis is conservative management with withdrawal of the causative agent and monitoring for improvement of renal function. Noncontrolled retrospective and observational studies have shown that there may be a role for immunosuppression with steroid treatment for cases in which the diagnosis of acute interstitial nephritis is confirmed by biopsy but kidney function does not improve after withdrawal of the suspected agent. ${ }^{3}$ Data from a recent retrospective study of acute interstitial nephritis suggest that early initiation of steroid therapy within 2 weeks of cessation of the causative agent may lead to improved renal function. ${ }^{5}$

Box 1: Common causes* of acute interstitial nephritis ${ }^{2,4,6}$

Drugs

- Antimicrobials (ampicillin, ciprofloxacin, methicillin, penicillin, rifampicin, sulfonamides)

- Nonsteroidal anti-inflammatory drugs (acetylsalicylic acid, fenoprofen, ibuprofen, indomethacin, naproxen, phenylbutazone, piroxicam, tolmetin, zomepirac)

- Acid suppressors (omeprazole, pantoprazole, rabeprazole, cimetidine)

- Others (phenytoin, furosemide, allopurinol, phenindione )

Infections

- Direct infiltration (leptospirosis, cytomegalovirus, candidiasis)

- Reactive to systemic infections (streptococcal infection, diphtheria, Hantavirus)

\section{Systemic diseases}

- Metabolic diseases (urate nephropathy, hypercalcemic nephropathy, oxalate nephropathy)

- Immunologic reactions (transplant rejection, systemic lupus erythematosis, sarcoidosis, cryoglobulinemia)

- Neoplastic diseases (lymphoproliferative diseases)

Idiopathic causes

*These are the most common causes of acute interstitial nephritis, but this list is not exhaustive.

\section{Acute interstitial nephritis and proton pump inhibitors}

The first case of acute interstitial nephritis caused by pantoprazole was reported in 2004. ${ }^{\prime}$ Acute interstitial nephritis has been described with multiple different proton pump inhibitors, including omeprazole, pantoprazole and rabeprazole. ${ }^{4,6}$ In the 2008 Canadian drug monograph for pantoprazole sodium, interstitial nephritis was listed as a reported adverse event in postmarketing use for which a causal relation to pantoprazole could not be ruled out. In 2007 in Canada, Pantoloc was the fourth most common out-patient prescription (5054 prescriptions dispensed), which was an increase of $16.3 \%$ from 2006. ${ }^{7}$ Although this adverse reaction is rare, the number of cases of acute interstitial nephritis induced by proton pump inhibitors is thought to be increasing because of their increasing widespread use. ${ }^{6}$ However, the exact rate of this rare adverse event is difficult to estimate because many patients are taking multiple medications, which also makes it difficult to establish a causal relation between proton pump inhibitors and acute interstitial nephritis.

In our patient's case, the most likely cause of his acute interstitial nephritis was pantoprazole. However, the occasional use of ibuprofen is another potential cause of acute interstitial nephritis. A rechallenge with pantoprazole was not performed because we felt that it would be unethical. Another hypothesis is that the use of ibuprofen in combination with pantoprazole increased the patient's susceptibility to developing acute interstitial nephritis through some unknown mechanism. A third potential cause was the daily use of acetylsalicylic acid, which can also cause acute interstitial nephritis. However, the patient had tolerated this medication daily for years. Because of the association of the onset of symptoms with pantoprazole therapy, the use of ibuprofen in response to the symptoms of acute renal failure experienced by the patient, and the longterm tolerance of acetylsalicylic acid, the most likely offending agent was pantoprazole.

Little data exists about approaches to treating acute interstitial nephritis caused by proton pump inhibitors. Thus, man-

Box 2: Characteristics of acute interstitial nephritis

- Acute elevation in creatinine levels (not caused by pre- or post-renal etiologies)

- General malaise, nausea (caused by buildup of metabolites)

- Normal blood pressure, no edema (distinguishes acute interstitial nephritis from acute tubular necrosis)

- Polyuria and polydypsia (kidneys unable to concentrate urine)

- Maculopapular rash (may be an early indication of drug-induced acute interstitial nephritis)

- Proteinuria (caused by tubular damage)

- Pyuria (occurs in almost all cases)

- Hematuria (occurs in about $90 \%$ of cases)

- Eosinophiluria (occurs in $80 \%$ of cases of drug-induced acute interstitial nephritis) 
agement should similar to that for general drug-induced acute interstitial nephritis. Because proton pump inhibitors are widely used, more studies are needed to understand the mechanism that leads to interstitial nephritis, the risk factors for its development and whether this is a class effect.

Because acute interstitial nephritis is a rare adverse effect of proton pump inhibitors, physicians should not hesitate to prescribe these drugs for appropriate indications in the absence of a history of acute interstitial nephritis. Rather, it is important that physicians be aware of the common drugs that can cause acute interstitial nephritis and monitor patients for the signs and symptoms of this disease because early intervention can improve the outcome. Finally, it is important to take a full drug history, including prescribed and over-thecounter medications, herbal remedies and illicit drugs, for patients who present with the signs and symptoms of acute renal failure.

This article has been peer reviewed.

Competing interests: None declared.

\section{REFERENCES}

1. Ra A, Tobe SW. Acute interstitial nephritis due to pantoprazole. Ann Pharmacother 2004;38:41-5.
2. Goldman L, Ausieloo D, editors. Cecil textbook of medicine. 22nd ed. Philadelphia (PA): Saunders; 2004.

3. Rossert J. Drug-induced acute interstitial nephritis. Kidney Int 2001;60:804-17.

4. Harmark L, van der Wiel HE, de Groot MC, et al. Proton pump inhibitor-induced acute interstitial nephritis. Br J Clin Pharmacol 2007;64:819-23.

5. Gonzalez E, Gutierrez E, Galeano C, et al. Early steroid treatment improves the recovery of renal function in patients with drug-induced acute interstitial nephritis. Kidney International 2008;73:940-6.

6. Brewster UC, Perazella MA. Proton pump inhibitors and the kidney: critical review. Clin Nephrol 2007;68:65-72.

7. IMS Health Canada. Top 50 dispensed medications in Canada, 2007. Montréal (Que): IMS Health Canada; 2008. Available: www1.imshealth.com/vgn/images /portal/cit_40000873/8/14/79014583Trends03_En07CORR.pdf (accessed 2009 Feb. 2).

The section Cases presents brief case reports that convey clear, practical lessons. Preference is given to common presentations of important rare conditions, and important unusual presentations of common problems. Articles start with a brief summary (100 words) outlining the case and its relevance to a general audience. The case presentation follows (500 words maximum) as well as a discussion of the underlying condition (1000 words maximum). Generally, up to 5 references are permitted and visual elements (e.g., tables of the differential diagnosis, clinical features or diagnostic approach) are encouraged. Written consent from patients for publication of their story is a necessity and should accompany submissions. See information for authors at www.cmaj.ca.

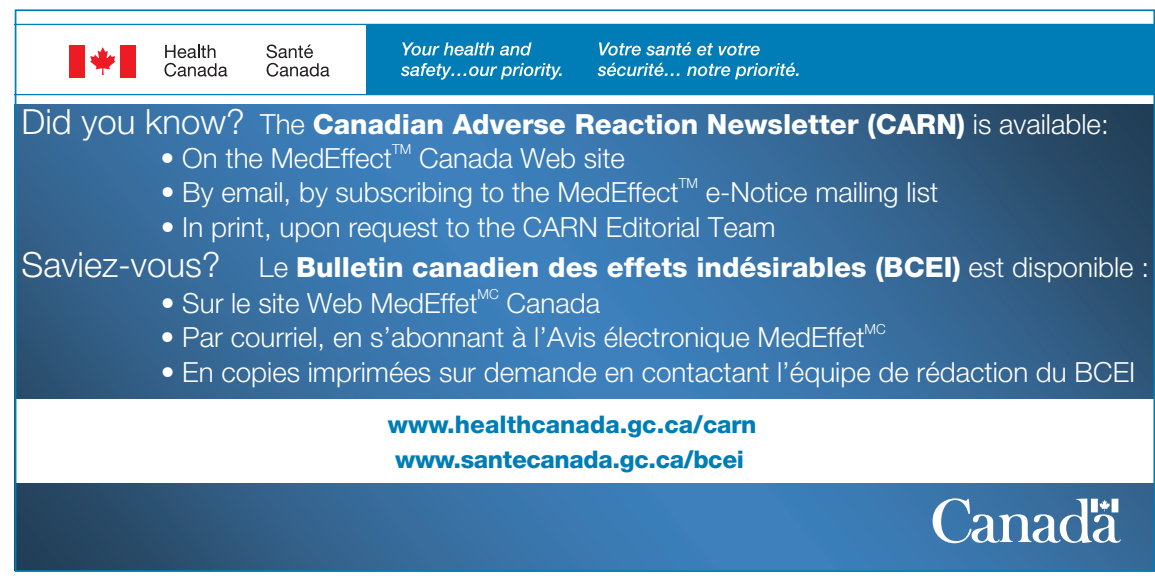

